Original article

\title{
Quality of life in patients with ischemic heart disease
}

\author{
Mohsen Taghadosi ${ }^{1,2}$, Zahra Aliakbarzade Arani ${ }^{3, *}$, Hamid Reza Gilasi ${ }^{4,5}$
}

(Received: 19 June 2013; Accepted: 17 Aug 2013)

\begin{abstract}
Background and Purpose: Ischemic Heart Diseases (IHD) is getting as the most common and the main causes of heart diseases and mortalities worldwide affecting the quality of life (QOL) of the patients. This study was conducted to determine the QOL for the patients with IHD referring to medical centers of Kashan in 2007.

Methods: This cross-sectional study was performed on all patients $(n=500)$ referring to the medical centers with definitive diagnosis of Myocardial Infarction (MI), having coronary artery disease, who have undergone percutaneous transluminal coronary angioplasty (PTCA) and coronary artery bypass graft (CABG) surgeries. QOL of the clients were measured respectively by short form-36 (SF-36) and Seattle angina questionnaire (SAQ) instruments and then scored as bad, medium, good, and very good based on the test scores. Demographic data and the major risk factors for cardiovascular diseases were also collected using the questionnaire. The data was analyzed by Chi-square and Fisher's exact tests along with Pearson's correlation coefficient and Kendall's rho.

Results: QOL based on the SF-36 and SAQ criteria were viewed with a mean of $112.5 \pm 24$ and 59.8 \pm 9.14 , respectively. Gender, older age, educational level, marital status, the number of children, residency location and having risk factor had influenced their life quality.

Conclusions: Correlation of the results between the two tests of SAQ and SF-36 and correlation between QOL levels have been positive and meaningful. The study offers more attention and education to the patients undergoing PTCA surgeries, the aged ones, patients with other diseases, singles, villagers, illiterate and female ones.
\end{abstract}

Keywords: Quality of life, Ischemic heart diseases, Myocardial infarction, Coronary artery bypass graft, Percutaneous transluminal coronary angioplasty

\section{Introduction}

Ischemic Heart Disease (IHD) as the most prevalent of heart diseases and the main cause of mortalities in the world today does affect patients' QOL (1). Reports on the prevalence or incidence of IHD in developing countries are very scarce, and routinely collected data is often incomplete and unreliable $(2,3)$. It is predicted that by 2020,25 million will be victims of cardiovascular diseases and they will be number-one cause of death and disability (4). These diseases are responsible for various for the patients and bring about considerable social costs (5). In MI patients, since the patients are facing decreased heart and physical capacity preceded by the ischemic heart attacks, as a result, the QOL is affected by this change (5-9). QOL is defined as the individual's unique cognition and a way to express feelings about his/ her health status (10). It indicates personal

\footnotetext{
${ }^{1}$ Department of Medical Surgical Nursing, Nursing and Midwifery School, Kashan University of Medical Sciences, Kashan, I. R. Iran. ${ }^{2}$ Ph.D Candidate, Department of Nursing, Medical Sciences School, Tarbiat Modares University, Tehran,I. R. Iran.

${ }_{3, *}{ }^{*}$ Corresponding author: Department of Operating room, Paramedical School, Qom University of Medical Sciences, Qom, IR. Iran. Email: zaliakbarzade@muq.ac.ir

${ }^{4}$ Department of Epidemiology, Public Health School, Shahid Beheshti University of Medical sciences, Tehran, IR. Iran.

${ }^{5}$ Department of Biostatistics and Epidemiology, Public Health School, Kashan University of Medical Sciences, Kashan, IR. Iran.
} 
perception of life in different aspects such as physical and psychosocial function which is in line with the patient's standards and expectations (11). Moreover, QOL is a good predictor of mortality and the need for hospitalization. It is also of great value in making decision regarding further procedures (12). In general, few studies conducted in relation to these cases show that QOL in heart failure is poorer compared with the other chronic diseases. In another study, the higher mean was related to mental health $(43.58 \pm 24.44)$ and the lower mean was associated with physical problem (5.87 \pm 21.11 ) (4). But there was no study comparing the QOL in different stages of heart disease.

A series of invasive strategies such as percutaneous transluminal coronary angiography (PTCA) and coronary artery bypass graft (CABG) are utilized to treat coronary heart diseases (1316). A study depicted that the patients undergoing CABG had a better and unchangeable QOL 6 and 12 months after the surgery compared with those experiencing PTCA 17 but the results of a research showed that the QOL of patients is low 3 months after CABG (18). Factors such as diabetes, number of angina attacks, receiving training, marital status, gender, education, insurance status, and the number of children have been recognized as the influencing factors on QOL (19-21). Another study revealed that the number of attacks influenced QOL (22). Since dropped QOL leads to depression, a sense of helplessness, danger of social harm and physical-mental inactivity (10), QOL has received much attention, accordingly. Due to the importance of QOL and the growing number of IHD patients and their QOL vagueness in Kashan, the present study has been performed to measure the QOL of the patients with IHD in 2007. The research results can be useful to diagnose patients suffering from heart problems with low QOL and thus to plan to allocate the necessary resources for their treatment and care.

\section{Materials and Methods}

This was a cross-sectional study. The study population included all of the patients referring to the medical centers of Kashan-based University of Medical Sciences for follow-up. After obtaining the necessary consent, the patients have been selected based on inclusion and exclusion criteria. We covered the individuals with definitive diagnosis of infarction (based on clinical and preclinical criteria) and chronic stable angina (based on angiography results). The study also included those who had PTCA and CABG, but are now under medical care showing no serious problem while it's been at least two months before their IHD has been started. They were conscious and inclined to answer the questions and they were not hospitalized 1-2 months before that. Patients with mental and acute illness were excluded from the study. SF-36 and SAQ instruments10, 13 have been used to measure QOL. SF36 is a 36item questionnaire measuring physical function, functional disorders and mental health due to emotional disorder and overall perception about health (23). SAQ questionnaire included 19 items in 5 separate parts including: physical activity limitations, persistent angina, recurrent angina, treatment status, and perception of the disease (10). According to SF-36, scores less than 38 denote low QOL. Scores 38-76 and 77-115 denote median and good QOL, respectively, while scores more than 115 imply very good QOL. According to SAQ, scores less than 30 denote low QOL, scores 31-50 and 51-70 respectively denote medium and good QOL, while scores more than 70 stand for very good QOL (24). In the study by McColl (2003), SAQ has been validated by alpha-Cronbach as 0.93 and SF-36 with $\alpha=0.88$ in role function and $\alpha=0.81$ in mental health (19). In the research on 20 unstable angina suffering patients in Kashan, SAQ got $\alpha=0.93$ and SF-36 
obtained $\alpha=0.90$. Demographic data along with the major risk factors for cardiovascular diseases (hypertension, diabetes, and hyperlipidemia) were collected by the questionnaire. Patients signed an informed consent after being provided with detailed information regarding the study goals. In addition, this research was approved by the Ethics Committee of the Research Deputy of Kashan Medical Sciences University. Confidentiality, anonymity, and the right of participants to withdraw from the study were presented. The data has been then analyzed using Chi-square and Fisher's exact tests along with Pearson's correlation coefficient and Kendall's rho.

\section{Results}

Out of 500 participants, $279(55.8 \%)$ were male and 221 (44.2\%) were female. The mean age of the patients was $60.5 \pm 12.5$ years. $60.3 \%$ of the patients had MI, 26.1\% had PTCA, 8.7\% had CABG, and $4.8 \%$ of them had chronic stable angina. In terms of occupation, $34.2 \%$ of the clients were housewives, $23.4 \%$ of them had paying jobs and the rest were workers, employees, and farmers. $65.8 \%$ of the patients had 4 children or more. In terms of education, $42.4 \%$ of the patients could hardly read and write. $38.4 \%$ of them were graduates and $22.2 \%$ were illiterate.

$30.6 \%$ of the participants had moderate QOL; $41.8 \%$ and $27.6 \%$ of them had good and very good QOL based on SAQ, respectively. $6.8 \%$ of the patients had moderate QOL while $45.4 \%$ and $47.8 \%$ of them had good and very good QOL based on SF36, respectively (Table 1).

Table 1. Relationship between Heart Diseases \& QOL based on SAQ \& SF36 Questionnaire

\begin{tabular}{|c|c|c|c|c|c|c|c|c|c|c|c|c|}
\hline \multirow{2}{*}{$\begin{array}{l}\text { QOL } \\
\text { Status }\end{array}$} & \multicolumn{6}{|c|}{ SAQ } & \multicolumn{6}{|c|}{ SF36 } \\
\hline & Moderate & Good & $\begin{array}{l}\text { Very } \\
\text { good }\end{array}$ & Total & $\begin{array}{l}\text { Mean } \\
(\mathrm{SD})\end{array}$ & P-value & Moderate & Good & $\begin{array}{l}\text { Very } \\
\text { good }\end{array}$ & Total & $\begin{array}{l}\text { Mean } \\
\text { (SD) }\end{array}$ & P-value \\
\hline \multirow[t]{2}{*}{$\mathrm{CABG}$} & 22 & 16 & 2 & 40 & 52.55 & $>0.001$ & 0 & 28 & 12 & 40 & 104.7 & $>0.001$ \\
\hline & (55) & (40) & (5) & (100) & (9.9) & & (0) & (70) & (30) & (100) & (18.7) & \\
\hline \multirow[t]{2}{*}{ PTCA } & 46 & 70 & 4 & 120 & 51.63 & & 22 & 62 & 36 & 120 & 99.94 & \\
\hline & (38.3) & (58.3) & (3.3) & (100) & (8.9) & & (18.3) & (51.7) & (30) & (100) & (20.6) & \\
\hline \multirow[t]{2}{*}{ MI } & 75 & 108 & 94 & 277 & 61.89 & & 9 & 118 & 150 & 277 & 116.41 & \\
\hline & $(27.1)$ & (39) & (33.9) & (100) & (14.9) & & (3.2) & (42.6) & (54.2) & (100) & (20.6) & \\
\hline \multirow[t]{2}{*}{ Angina } & 10 & 15 & 38 & 63 & 59.73 & & 3 & 19 & 41 & 63 & 109.97 & \\
\hline & (15.9) & (23.8) & (60.3) & (100) & (13.5) & & (4.8) & (30.2) & (65.1) & (100) & (25.8) & \\
\hline \multirow[t]{2}{*}{ Total } & 153 & 209 & 138 & 500 & 58.29 & & 34 & 277 & 399 & 500 & 110.77 & \\
\hline & (30.6) & (41.8) & (27.6) & (100) & (13.9) & & (6.8) & (45.4) & (47.8) & (100) & (23.7) & \\
\hline
\end{tabular}

Information about the relationship between patients' QoL and most of the dependent variables has been presented in Table 2. 
Table 2. Factors associated with quality of life of heart disease patients based on SAQ \& SF36 questionnaire

\begin{tabular}{|c|c|c|c|c|c|c|c|c|c|c|}
\hline \multirow[t]{2}{*}{ QOL } & \multicolumn{5}{|c|}{ SAQ } & \multicolumn{5}{|c|}{ SF36 } \\
\hline & \multicolumn{9}{|c|}{ Effective Factor } & P-value \\
\hline Age & & & & & $<0.001$ & & & & & $<0.001$ \\
\hline \multirow[t]{3}{*}{$\leq 50$} & 29 & 50 & 76 & 155 & & 5 & 50 & 100 & 155 & \\
\hline & (18.7) & $(32.2)$ & (49) & $(100)$ & & (3.2) & $(32.2)$ & (64.5) & (100) & \\
\hline & 124 & 159 & 62 & 345 & & 29 & 177 & 139 & 345 & \\
\hline$>50$ & (35.9) & (46) & (17.9) & (100) & & (8.4) & $(51.3)$ & $(40.2)$ & (100) & \\
\hline Gender & & & & & $=0.016$ & & & & & $=0.045$ \\
\hline \multirow[t]{3}{*}{ Male } & 100 & 109 & 70 & 279 & & 19 & 140 & 120 & 279 & \\
\hline & (35.8) & (39.1) & (25.1) & (100) & & (6.8) & $(50.2)$ & (43) & (100) & \\
\hline & 53 & 100 & 68 & 221 & & 15 & 87 & 119 & 221 & \\
\hline Female & (24) & $(45.2)$ & $(30.8)$ & (100) & & $(6.8)$ & (39.4) & $(53.8)$ & 100 & \\
\hline Education & & & & & $=0.311$ & & & & & $=0.036$ \\
\hline \multirow{3}{*}{ literate } & 113 & 164 & 112 & 389 & & 22 & 171 & 196 & 389 & \\
\hline & (29) & (42.2) & (28.8) & (100) & & (5.6) & (43.9) & (50.3) & (100) & \\
\hline & 40 & 45 & 26 & 111 & & 12 & 56 & 43 & 111 & \\
\hline illiterate & (36) & $(40.5)$ & (23.4) & (100) & & (10.8) & $(50.5)$ & (38.7) & (100) & \\
\hline Marital & & & & & $<0.001$ & & & & & $=0.002$ \\
\hline \multirow[t]{2}{*}{ single } & 17 & 2 & 13 & 32 & & 4 & 15 & 13 & 32 & \\
\hline & (53.1) & $(6 / 2)$ & (40.6) & (100) & & (6.8) & (46.8) & (40.6) & (100) & \\
\hline \multirow{2}{*}{ married } & 136 & 207 & 125 & 468 & & 30 & 212 & 226 & 468 & \\
\hline & (29.1) & $(44.2)$ & (26.7) & (100) & & (6.4) & $(45.3)$ & (48.3) & (100) & \\
\hline $\begin{array}{l}\text { Number of } \\
\text { children }\end{array}$ & & & & & $<0.001$ & & & & & $<0.001$ \\
\hline \multirow{2}{*}{$3 \geq$} & 38 & 61 & 72 & 171 & & 6 & 55 & 110 & 171 & \\
\hline & (22.2) & (35.6) & (42.1) & (100) & & (3.5) & $(32.2)$ & $(64.3)$ & (100) & \\
\hline \multirow{2}{*}{$3<$} & 115 & 148 & 66 & 329 & & 28 & 172 & 129 & 329 & \\
\hline & (35) & (45) & (20) & (100) & & (8.5) & (52.3) & $(39.2)$ & (100) & \\
\hline Place of residence & & & & & $=0.172$ & & & & & $=0.044$ \\
\hline \multirow[t]{2}{*}{ City } & 116 & 164 & 107 & 387 & & 28 & 170 & 189 & 387 & \\
\hline & (30) & $(42.4)$ & (27.6) & (100) & & $(7.2)$ & $(43.9)$ & (48.9) & $(100)$ & \\
\hline \multirow{2}{*}{ Village } & 22 & 29 & 10 & 61 & & 2 & 37 & 22 & 61 & \\
\hline & (36.1) & $(47.5)$ & (16.4) & (100) & & (3.3) & (60.6) & $(36.1)$ & (100) & \\
\hline Risk factors & & & & & $<0.001$ & & & & & $<0.001$ \\
\hline \multirow[t]{3}{*}{$2 \geq$} & 83 & 169 & 137 & 389 & & 13 & 145 & 231 & 389 & \\
\hline & (21.3) & (43.4) & $(35.2)$ & (100) & & (3.3) & $(37.3)$ & (59.4) & (100) & \\
\hline & 70 & 40 & 1 & 111 & & 21 & 82 & 8 & 111 & \\
\hline $2<$ & $(63.1)$ & (36) & $(0.9)$ & (100) & & $(18.9)$ & $(73.9)$ & $(7.2)$ & (100) & \\
\hline
\end{tabular}

The results correlation between the two tests of SAQ and SF-36 with $\mathrm{r}=0.867$ (Pearson) and P-value $=0.001$ and the correlation between the levels of QOL (Moderate, Good, Very Good) with $\mathrm{r}=0.725$ (Spearman) and P-value $=0.001$ were positive and statistically significant. The correlation coefficient of Kendall's rho was 0.68 and meaningful. Kappa index was at 0.033 and significant (Table 3). 
Table 3. Agreement on the quality of life for heart disease patients based on SAQ \& SF36 Questionnaire

\begin{tabular}{|c|c|c|c|c|}
\hline \multicolumn{5}{|l|}{ SAQ } \\
\hline \multirow[t]{2}{*}{ Moderate } & 29 & 116 & 8 & 153 \\
\hline & (5.8) & (23.2) & (1.6) & (30.6) \\
\hline \multirow[t]{2}{*}{ Good } & 5 & 111 & 93 & 209 \\
\hline & (1.0) & $(22.2)$ & (18.6) & (41.8) \\
\hline \multirow{3}{*}{ very good } & 0 & 0 & 138 & 138 \\
\hline & (0) & (0) & (27.6) & (27.6) \\
\hline & 34 & 227 & 239 & 500 \\
\hline Total & (6.8) & $(45.4)$ & (47.8) & (100) \\
\hline
\end{tabular}

\section{Discussion}

Chronic heart failure is a serious syndrome that affects the functional status of those patients suffering from it. It also seriously limits their activities and influences their everyday living. The findings of this study demonstrated that QOL was mostly good and $93.2 \%$ of the patients with IHD had good and very good QOL based on SF-36; in none of the patients bad QOL was observed. QOL in IHD patients was low in those with $\mathrm{MI}$, chronic stable angina, $\mathrm{CABG}$, and PTCA seen in descending order. In other words, the mean score of QOL in the patients with MI stood at the highest rank, whereas in the patients having PTCA, it stood at the lowest level based on SAQ criterion; similar results were obtained according to SF-36 criterion. Results in the study by David et al, (2011) also showed that based on SAQ, the mean QOL in CABG patients $(69.5 \pm 26.7 / \mathrm{n}=897)$ was better than that of PCI patients $(45.5 \pm 24.7 / \mathrm{n}=903) 25$. Also the study performed by Saldana (2003) indicated that QOL was $76.4 \pm 14.5$ two months after angioplasty. The findings demonstrated that individuals undergoing CABG surgery have improved QOL comparing with those experiencing PTCA surgery (P-value=0.001). The difference was more significant based on SF-36 than that on SAQ and Sf-36 showed higher QOL in patients under CABG16.Difference in the result may be due to difference in sample size. By using SAQ criteria, Borken et al, (2002) analyzed the health status of 252 and 223 individuals experiencing PTCA and $\mathrm{CABG}$ surgeries, respectively up to 6 and 12 months following the procedures. They concluded that $25 \%$ and $1 \%$ of the individuals who had PTCA and $\mathrm{CABG}$, respectively, required percutaneous coronary intervention (PCI). Their findings indicated that angina recovers much better after CABG surgery than PTCA surgery $(\mathrm{P}<0.001) 17$.Rumsfeld (2003) performed a comparative study on QOL and health between drugresistant patients with IHD at high risk after PCI and those patients having experienced CABG surgery. The results revealed that the individuals undergoing PCI were probably more prone to angina and had to undergo repeated PCI procedure (26).

The present study confirms that the number of risk factors (hypertension, diabetes, and hyperlipidemia) for the patients with IHD increases their QOL according to SAQ and SF -36 criteria. Baghiani Moghadam in a study on the correlation of diabetes patients'QOL and risk factors for cardiovascular diseases gained the same findings (27).The Correlation of education level with SAQ criterion $(\mathrm{P}$-value $=0.311)$ was not significant while the correlation based on SF-36 (P-value $=0.033$ ) was meaningful. The higher education level, the more enhanced QOL scores. Education has been always influencing health and disease and the other aspects of life by making essential changes in the individuals' attitude and insight. Rahnavard et al, (2006) in the study on QOL and its influencing factors in heart disease patients showed that there was a meaningful relationship between education level and QOL (28). The relationship between marital status and QOL was significant according to SAQ and SF-36 criteria (P-value $=0.001 \&$ P-value $=0.002$, respectively), so that the individuals with medium QOL were mostly widows or the divorced ones. Married and single participants had good and very good QOL. Hasanpour (2007) (29) and Rahnavard (2006)(28) reported no meaningful correlation between marital status and QOL. On the contrary, Simpson (2005)(30) concluded that there was a relationship between marital status 
and QOL. Luttik (2005) stated that supporting sources are important for the survival and compatibility of heart disease patients living alone with low QOL(31). The number of children had a significant correlation with SAQ and SF-36 criteria (living alone=0.001) so that increase in the number of children resulted in the patient's QOL decrease. It seems that the increase in the number of children in communities with low incomes due to economic limitation and inadequate medication can contribute to low QOL which deserves further investigation. Residency location had negative correlation with QOL based on SAQ (P-value=0.172), but showed a positive correlation based on SF-36. Individuals living in the cities had more elevated QOL than those residing in the villages ( $\rho$-value $=0.044)$. QOL appeared to be significantly higher in women than in men based on SAQ and SF-36 criteria $(\rho$-value $=0.016$ and $\rho$-value $=0.045)$. However, Reynolds (2006) (32) and Howes (2001) (33) reported that QOL was meaningfully lower in women. Cline proved that QOL was higher in men with heart failure than in women (34). According to Riedinger (2002) QOL in men in terms of physical and social aspects is specifically higher than that in women. He also stated that since women do exercises much less than men, consequently, this weakens their functional and physical activities and leads to low QOL (35). The difference in this study finding in terms of QOL by gender with those of the other studies is not known to the authors; therefore, more comprehensive studies are required in this regard. The study Findings implied that QOL decreases significantly as the participant gets older. Kovacik (2012) (36), Reynold (2006) (32), and Howes (2001) (33) pointed out the same correlation in their findings. However, Hatamipour (2005) (28), Rahnavard (2006) (28), and Hasanpour (2007) (29) found no significant correlation between age and QOL.

? Agreement without the chance of $0 / 033$ indicates moderate agreement in SAQ and SF-36. Saldana (2003) also concluded that SAQ was a highly accredited and sensitive instrument to measure QOL (16). Dempster (2000) in the study to measure health- related QOL in IHD patients stated that SF-36 is the most reliable and sensitive way to measure QOL. He also claimed that there are 10 instruments to measure QOL for IHD patients among which SAQ or Seattle Angina Questionnaire is regarded as the most important one (37). Seki et al, (2010) in their study calculated QOL correlation in heart patients based on Seattle angina questionnaire, Japanese version (SAQ-J) \& SF36 questionnaire. The QOL ranges of SAQ-J were moderately correlated with Role Functioning-Emotional ranges than the other ones in SF36, which were expected to have higher correlation (38). Missing the medical background and forgetting some information about the patients are the limiting factors influencing the result of our study that was compensated by increasing the sample size.

\section{Conclusion}

Our findings revealed that QOL of patients undergoing IHD surgery based on SF-36 was in the range of good and medium whereas it was mostly good and very good according to SAQ. According to moderate agreement in these two instruments and the previous study results, SAQ and SF-36 are the most appropriate tools to assess quality of life in heart disease patients. The patients with a history of CABG had better quality of life than those undergoing PTCA. Gender, older age, educational level, marital status, the number of children, residency and having risk factor have influenced their quality of life. Therefore, more attention should be paid to the patients undergoing PTCA surgeries, the elderly, and the patients with other diseases, singles, villagers, illiterate and female ones.

\section{Conflict of interests}

The authors declare that they have no competing interests.

\section{Author's contributions}

M.Taghadosi have contributed to the design,has written the draft, Hr.Gilasi performing statistical 
analysis and contributed to design, Z.Aliakbarzade arani interpreting the discussion, revising the content, and approving the final manuscript as well.

\section{Acknowledgement}

We appreciate research deputy of Kashan Medical Sciences University and all of the patients participating in this study.

\section{References}

1. Smettzer S.C, Bare B.J, Hinkle J.L, Cheever K.H, Brunner and suddarth. Text book of Medical Surgical Nursing. 11th ed. Philadelphia: Lippincott Williams Company; 2008.

2. Mandal S, Saha JB, Mandal SC, Bhattacharya RN, Chakraborty M, Pal PP. Prevalence of Ischemic Heart Disease among Urban Population of Siliguri, west Bengal. Indian J Community Med 2009; 34(1): 19-23.

3. Suliman A. The State of Heart disease in Sudan. Cardiovasc J Afr 2011; 22(4): 191-6.

4. Yousefi P, Sabzevari S, Mohammad Alizadeh S, Haghdoost A. Studying life quality in Heart Failure Hospitalized Patients in Kerman Medical University Hospital in 2008. J Nurs Res 2011; 6(21): 59-67 (Persian).

5. Phipps W.J, Marek J.E, Manahan F.D, Neighbor S, Sands J.K, Green OJ. Medical- Surgical Nursing. Health and Illness Perspectives. 7th ed. Missouri: Mosby publication; 2003.

6. Azadi F, Mohammadi A the Effect of Using Cooperative Care Model on the QOL of Coronary Artery Disease Patients. Nurs Res 2006; 1(2): 23-29 (Persian).

7. Shidfar Mshojaeizade D, Hossini M, Asasi N, Majlesi F, Nazemi S. Surveying Knowledge, Attitude and Life Style in Patients with Unstable Angaina, Mashhad University of Medical sciences Hospital. Payesh Health Monit 2002; 7(2):49-61 (Persian).

8. Goldman L, Claude Bennet J. Cecil Text Book of Medicine. 21th ed. W.B. Saunders Company: USA, 2000.

9. Rooh Afza H, Saeidi M, Sadegi M, Bashtam M, Rbiee K. Effects of a cardiac rehabilitation course on psychological stresses in an Iranian population. ARYA Atheroscler 2012: 7(Suppl): 74-77.
10. Gill TM, Feinstein AR. A critical Appraisal of Qualityof-Life Measurements. JAMA 1994; 272(8): 619-26.

11. Saeidi M, Mostafavi S, Heidari H, Masoudi S. Effects of a Comprehensive Cardiac Rehabilitation Program on Quality of Life in Patients with Coronary Artery Disease. ARYA Atheroscler 2013; 9(3):179-185.

12. Papadopoulou EF, Mavrogeni SI, Dritsas A, Cokkinos DV. Quality of Life Assessment Using Three Activity Questionnaires in Heart Failure Patients After Monthly, Intermittent Administration of Levosimendan During a SixMonth Period. Hellenic J Cardiol 2009; 50 (4): 269-274.

13. Smith HJ, Taylor R, Mitchell A. A Comparison of Four QOL Instruments in Cardiac Patients: SF-36, QLI, QLMI, and SEIQoL. Heart 2000; 84(4): 390-4.

14. Taylor C, Li lis C, Lemon P. the Art \& Science of Nursing Care. The Fundamentals of Nursing. Philadelphia: Lippincott 2001: 708-710.

15. Eugene B, Anthony S. F, Dennis L. K, Stephen L. U, Danl L, Longo J, Larry J. Harrisons Principles of Internal Medicine . 15 th ed. New Delhi: The McGrawHill companies; 2001.

16. Saldana G, Soloria S. Coronary Artery Angioplasty QOL Seattle angina Questionnaire. Rev max cardial 2003; 14(3): 94-97.

17. Borkon AM, Muehlebach GF, House J, Marso SP, Spertus JA. A Comparison of the Recovery of Health Status after Percutaneous Coronary Intervention and Coronary Artery Bypass. Ann Thorac Surg 2002; 74(5):1526-30.

18. Bahramnezhad F, Asadi Noughabi AA, Sief H, Mohammadi Y. Quality of life in Patients with Coronary Bypass Graft. Iran J Nurs Res 2012; 7(26):34-41 (Persian).

19. McColl E, Eccles MP, Rousseau NS, Steen IN, Parkin DW, Grimshaw JM. From Generic to ConditionSpecific? Instrument Order Effects in QOL Assessment. Med Care 2003; 41(7):777-90.

20. Mayo R, Bryant B, Turner R. QOL in NIDDM and A Comparison with IDDM. J of Psychosomatic Research1990; 34(1): 1-11.

21. Hunt Jo, Hendrata MV, Myles PS. QOL 12 Months after Coronary Artery Bypass Graft surgery. Heart and lung 2000; 29(6):401-411. 
22. John A, Adam C, Philip G, Darcy Green Conaway, Randall C. Predictors of Quality-of-Life Benefit After Percutaneous Coronary Intervention. Circulation 2004;110:3789-3794.

23. West RR, Jones DA, Henderson AH. Rehabilitation after myocardial infarction trial (RAMIT): Multicentre Randomized Controlled Trial of Comprehensive Cardiac Rehabilitation in Patients Following Acute Myocardial Infarction. Heart 2012 Apr; 98(8):637-44.

24. Dehkordi AH, Nazari AA, Heidar-Nejad MS, AsadiNoghabi AA, Forouzandeh N, Kasiri KA, Masoudi R, Mousavi A, Hosseini SM. Factors Influencing Quality of Life in Patients with Myocardial Infraction. Iran Journal of Nursing (IJN) 2009; 22(57): 43-52 (Persian).

25. Cohen DJ, Hout BV, Serruys PW, Mohr FW, Carlos Macaya C, Heijer P, Vrakking M, Wang K, et al. Quality of Life after PCI with Drug-Eluting Stents or CoronaryArtery Bypass Surgery. N Engl J Med 2011; 364(11): 1016-26.

26. Rumsfeld JS, Magid DJ, Plomondon ME, Sacks J, Henderson W, Hlatky M, Sethi G, Morrison DA. HeartRelated QOL after percutaneous Coronary Intervention Versus CABB in High-Risk Patients with Medically Refractory Ischemia. J Am Coll Cardiol 2003 May 21; 41(10):1732-8.

27. Bagiani Mogaddam M, Afkhami Ardacani M, Mazlomi S, Saeidizade M. Survay on the QOL of Diabetes Type 2 Patients. . J Shaheed Sadoughi Univ Med Sci 2006; 4(14):49-54 (Persian).

28. Rahnavard Z, Zolfaghari M, Kazemnejad A, Hatamipour Kh. An Investigation of QOL \& Factors Affecting It in Patients with Congestive Heart Failure. HYAT. 2006; 1(12): 77-86 (Persian).

29. Hasanpour-Dehkordi A, Delaram M, Foruzandeh N, Ganji F, Asadi Noghani AA, Bakhsha F, Sadegi B. A Survey on QOL in Patients with Myocardial Infarction, Referring to Shahrekord Hagar Hospital in 2005. Shahrekord University of Medical Sciences J 2007; 9(3): 78-84 (Persian)

30. Simpson E, Pilote L. Quality of life after Acute
Myocardial Infarction: A Comparison of Diabetic Versus Non-Diabetic Acute Myocardial Infarction Patients in Quebec Acute Care Hospital. Health Qual life outcomes 2005; 3: 80 .

31. Luttik ML, Jaarsma T, Veeger NJ, van Veldhuisen DJ. For better and for worse: Quality of life impaired in HF patients as well as in their partners. Eur J Cardiovasc Nurs. 2005 Mar; 4(1): 11-14.

32. Reynolds MR, Lavelle T, Essebage V, Cohen DJ, Zimetbaum P. Influence of Age, Sex, and Artial Fibrillation Recurrence on Quality of Life Outcomes in a Population of Patients with New-Onset Artrial Fibrillation: the Fibrillation Registry Assessing Costs, Therapie, Adverse Events \& Lifestyle (FRACTAL) Study. Am Heart J 2006 Dec; 152(6):1097-103.

33. Howes CJ, Reid MC, Brandt C, Ruo B, Yerkey MW, Prasad B, et al. Exercise Tolerance and Quality of Life in Elderly Patients with Chronic Atrial Fibrillation. J cardiovasc pharmacol ther 2001; 6(1): 23-29.

34. Cline EM, Willenheimer RB, Erhardt LR, Wiklund I, Israelsson BY. Health Related Quality of Life in Elderly Patients with Heart Failure. Scand cardiovascy 1999; 33(5): 278-285.

35. Riedinger MS, Dracup KA, Brecht ML. Quality of life in Women with Heart Failure 'Normal Groups and Patients with Chronic Condition. American Journal of Cridical Care. Heart Lung 2002; 11 (3): 211-219.

36. Kovacik M, Madarasz S, Kral M, Veverka T, Herzig R, Kanovsky P. Risk factors Associated with Ischemic Heart Disease Occurrence in Acute Ischemic Stroke Patients. Biomed Pap Med Fac Univ Palacky Olomouc Czech Repub 2013, 157(2):168-171.

37. Dempster M, Donnelly M. Measuring Health Related QOL of People with Ischemic Heart Disease. Heart 2000; 83(6):641-644.

38. Seki S, Kato N, Ito N, Kinugawa K, Ono M, Motomura $\mathrm{N}$, et al. Validity and Reliability of Seattle Angina Questionnaire (Japanese Version) in Patients with Coronary Artery Disease. Asian Nurs Res 2010; 4(2): 57-63. 